Draft VERSion June 25, 2018

Preprint typeset using $\mathrm{LAT}_{\mathrm{E}} \mathrm{X}$ style emulateapj v. 11/12/01

\title{
DYNAMICAL EXPANSION OF IONIZATION AND DISSOCIATION FRONT AROUND A MASSIVE STAR : A STARBURST MECHANISM
}

\author{
TAKashi Hosokawa ${ }^{1}$ And Shu-IChIRo InUtsuka ${ }^{2}$ \\ Draft version June 25, 2018
}

\begin{abstract}
We quantitatively examine the significance of star formation triggered in the swept-up shell around an expanding $\mathrm{H}$ II region. If the swept-up molecular gas is sufficiently massive, new OB stars massive enough to repeat the triggering process will form in the shell. We determine the lower limit $\left(M_{\mathrm{thr}}\right)$ for the mass of the star that sweeps up the molecular gas, where at least one new star with mass $M_{*}>M_{\text {thr }}$ forms after the shell fragmentation. To calculate the threshold stellar mass, $M_{\mathrm{thr}}$, we examine how massive molecular shells can form around various central stars, by performing detailed numerical radiation hydrodynamics calculations. The mass of the photodissociated gas is generally larger than the mass of the photoionized gas. However, the swept-up molecular mass exceeds the photodissociated mass with a higher-mass star of $M_{*} \gtrsim 20 M_{\odot}$. The accumulated molecular mass generally increases with the stellar mass, and amounts to $10^{4-5} M_{\odot}$ for $M_{*} \gtrsim 20 M_{\odot}$ with an ambient density of $n \sim 10^{2} \mathrm{~cm}^{-3}$. The threshold stellar mass is $M_{\mathrm{thr}} \sim 18 M_{\odot}$ with the star-formation efficiency of $\epsilon \sim 0.1$ and $n \sim 10^{2} \mathrm{~cm}^{-3}$. We examine the generality of this mode of run-away triggering for different sets of parameters, and found that $M_{\mathrm{thr}} \sim 15-20 M_{\odot}$ in various situations. If the ambient density is too high or the star-formation efficiency is too low, the triggering is not run-away, but a single event.
\end{abstract}

Subject headings: Circumstellar matter - H II regions - ISM: molecules - STARS : formation

\section{INTRODUCTION}

In galaxies, most of the stars are born in localized active star-forming regions or starburst regions. Clustered star formation is the dominant mode in these regions (e.g., Evans 1999; Lada \& Lada 2003), and several star clusters, including OB stars, form over a few Myrs from the parental giant molecular cloud (Williams \& McKee 1997). The newly born massive stars promptly emit the UV $(h \nu>13.6 \mathrm{eV})$ and far-UV (FUV $; 11 \mathrm{eV} \lesssim h \nu<13.6 \mathrm{eV})$ radiation, and the radiative feedback by these photons occurs much earlier than the supernova explosions. The UV and FUV radiation from massive stars has two competing effects on the parental molecular cloud. One is the negative feedback effect on the star formation activity. The parental molecular cloud is ionized and heated up by the UV radiation, and the nearby star formation is quenched (e.g., Whitworth 1979; Franco et al. 1994; Williams \& McKee 1997; Matzner 2002). Diaz-Miller et al. (1998) have shown that the photodissociation by FUV photons is more significant than the photoionization by UV photons. The other is the positive feedback effect. The next star formation is triggered in the compressed dense layer around the H II region (collect and collapse scenario ; e.g., Elmegreen \& Lada 1977; Elmegreen 1989). Recent observations have provided dramatic snapshots of this triggering process (e.g., Deharveng et al. 2003, 2005; Zavagno et al. 2005). Keto, Ho \& Lo (2005) have observed details of the starburst regions, and suggested that the propagating star formation is important on the scale of the clouds. The character of star formation depends on which feedback effect dominates the other in the cloud.

In our previous papers, Hosokawa \& Inutsuka (2005, 2006) (hereafter Papers I and II), we have studied the time evolution of the H II region, photodissociation re- gion (PDR), and the swept-up shell, performing detailed numerical calculations. We have shown that the molecular gas is accumulated around the H II region, and that the gravitational fragmentation of the shell is expected in many cases with the homogeneous molecular ambient medium. This suggests that the expanding H II region is an efficient trigger of the star formation in the cloud. In this letter, we examine the net feedback effect of the expanding H II region and PDR around a massive star.

\section{A STARBURST MECHANISM}

\subsection{Triggering Threshold Condition}

First, we focus on the positive feedback process for triggering the star formation in the shell. Let us consider a situation where a dense molecular shell, whose mass is $M_{\mathrm{sh}, \mathrm{m}}$, forms around a massive star, and the subsequent star formation is triggered after fragmentation of the shell. The total number of newly born stars is calculated as

$$
N_{*}=\frac{M_{\mathrm{sh}, \mathrm{m}} \epsilon}{M_{\mathrm{av}}}
$$

where $\epsilon$ is the star formation efficiency (SFE) for the swept-up gas and $M_{\mathrm{av}}$ is the average stellar mass, which is defined as,

$$
M_{\mathrm{av}} \equiv \int_{0}^{\infty} \phi(M) M d M / \int_{0}^{\infty} \phi(M) d M,
$$

where $\phi(M)$ is the initial mass function (IMF). Below, we use the IMF by Miller \& Scalo (1979). The calculated value of $M_{\mathrm{av}}$ is about $0.6 M_{\odot}$. The mass of the swept-up molecular gas, $M_{\mathrm{sh}, \mathrm{m}}$, generally depends on the ambient number density and mass of the central star (see Paper II). If $M_{\mathrm{sh}, \mathrm{m}}$ is sufficiently large, another massive star as well as lower-mass stars will form in the shell. Another

${ }^{1}$ Division of Theoretical Astrophysics, National Astronomical Observatory, Mitaka, Tokyo 181-8588, Japan ; hosokawa@th.nao.kyoto-u.ac.jp

2 Department of Physics, Kyoto University, Kyoto 606-8502 ; inutsuka@tap.scphys.kyoto-u.ac.jp 
H II region expands around this newly born massive star, and the triggering process will repeat. Furthermore, if the number of newly-born stars is larger than that of the previous generation, this process causes run-away triggering or a burst of star formation.

In order to examine the efficiency of this mode of triggering, we presume that the run-away triggering begins with a star more massive than the threshold stellar mass, $M_{\mathrm{thr}}$. A star of $M_{\mathrm{thr}}$ produces a shell that barely generates just one star more massive than $M_{\mathrm{thr}}$. If the triggering cycle begins with a star of $M_{*}>M_{\mathrm{thr}}$, multiple stars of $M_{*}>M_{\mathrm{thr}}$ form as "second generation" stars. The number of newborn stars increases as the triggering cycle advances, and the burst of star formation occurs. We represent the condition for the run-away triggering with simple equations. The number of $M_{*}>M_{\mathrm{thr}}$ stars born in the shell is,

$$
N_{\mathrm{thr}}=f_{\mathrm{thr}} N_{*}
$$

where $f_{\mathrm{thr}}$ is the number ratio of $M_{*}>M_{\mathrm{thr}}$ stars calculated as,

$$
f_{\mathrm{thr}} \equiv \int_{M_{\mathrm{thr}}}^{\infty} \phi(M) d M / \int_{0}^{\infty} \phi(M) d M
$$

We can determine $M_{\text {thr }}$ from the triggering threshold condition; $N_{\mathrm{thr}}=1$. Combining this condition and equations (1) and (3), we obtain

$$
M_{\mathrm{sh}, \mathrm{m}}=\frac{M_{\mathrm{av}}}{\epsilon f_{\mathrm{thr}}} .
$$

Evaluating how $M_{\text {sh,m }}$ depends on the stellar mass, we can calculate $M_{\text {thr }}$ for a given ambient number density and SFE by equation (5). If the molecular mass of the shell is smaller than $M_{\mathrm{av}} / \epsilon f_{\mathrm{thr}}$, triggering is not run-away, but only a single event. We examine $M_{\mathrm{sh}, \mathrm{m}}$ with various stellar masses and ambient number densities based on numerical calculations.

\subsection{Mass of the Molecular Shell v.s. Mass of the Central Star}

In order to evaluate the threshold stellar mass, $M_{\text {thr }}$ for the burst of star formation, we examine how the shell mass, $M_{\mathrm{sh}, \mathrm{m}}$ changes with the mass of the massive star and the ambient molecular gas density. Even if the shell forms around the H II region, the accumulated gas is exposed to FUV radiation from the central star. Unless the FUV radiation is blocked, almost no cold molecular gas accumulates in the shell. Therefore, we should carefully estimate the mass of the cold molecular gas in the shell, which is available for the next star-formation episode. For this purpose, we have calculated the time evolution of the $\mathrm{H}$ II region, PDR, and the swept-up shell around various central stars in the homogeneous molecular medium (see Paper II for the details). In this subsection, we focus on the fiducial case with $n_{\mathrm{H}}=100 \mathrm{~cm}^{-3}\left(n_{\mathrm{H}_{2}}=50 \mathrm{~cm}^{-3}\right)$. If the FUV radiation is efficiently shielded in the shell, the accumulated molecular mass, $M_{\mathrm{sh}, \mathrm{m}}$ increases as the H II region expands. However, $M_{\mathrm{sh}, \mathrm{m}}$ is actually limited by the following two factors:

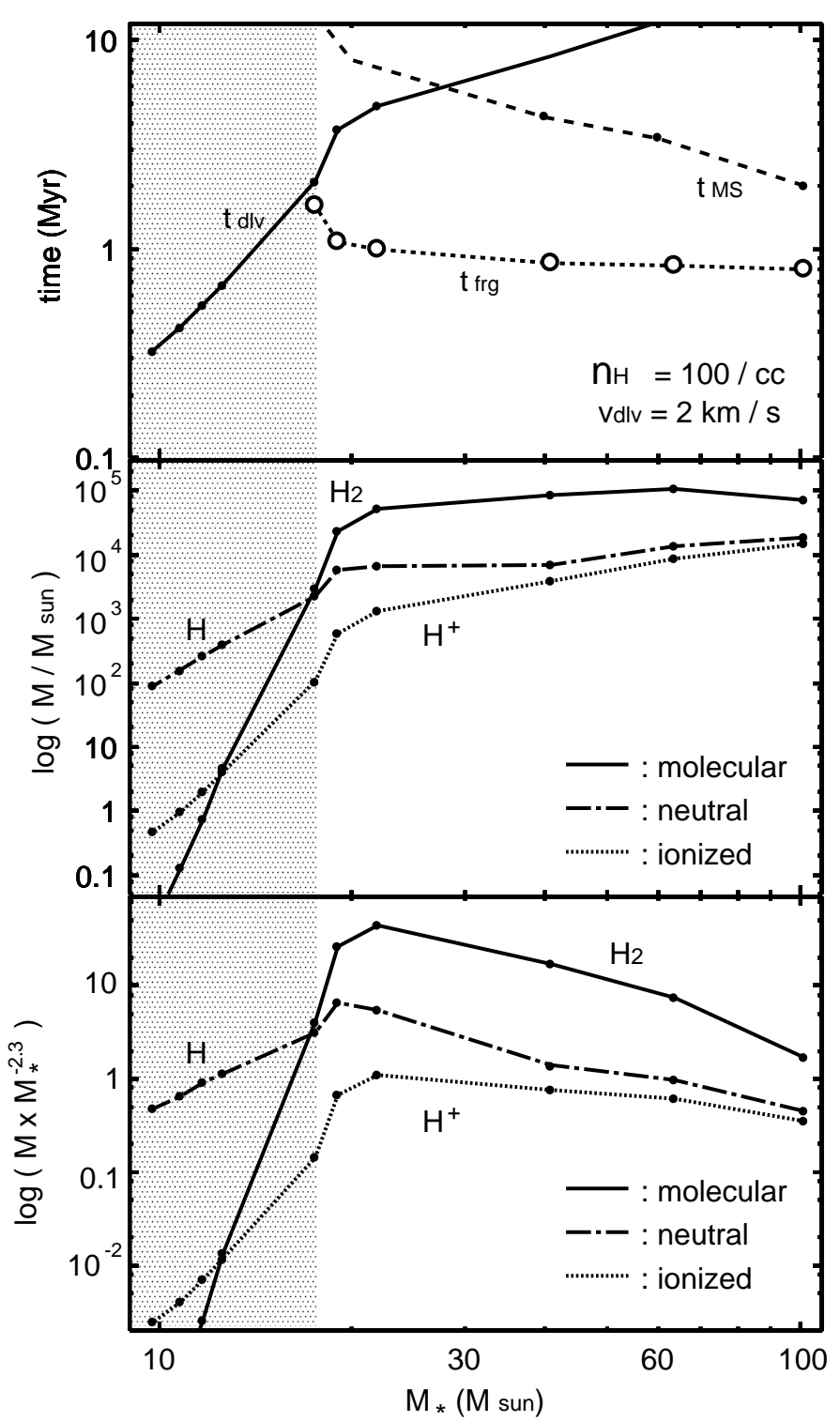

FIG. 1.- Top panel : Stellar mass v.s. the termination time of the shell in the ambient medium of $n_{\mathrm{H}}=100 \mathrm{~cm}^{-3}$ with the dissolving velocity, $v_{\mathrm{dlv}}=2 \mathrm{~km} / \mathrm{s}$. The solid and broken lines represent the dissolving time of the shell, $t_{\mathrm{dlv}}$ and the main-sequence lifetime of the central star, $t_{\mathrm{MS}}$ respectively. The termination time of the shell is the smaller one between $t_{\mathrm{dlv}}$ and $t_{\mathrm{MS}}$. The dotted line means the time when the gravitationally unstable region appears in the shell. The dots and open circles indicate the calculated values in each model. Middle panel : The mass of molecular hydrogen accumulated in the shell (solid line), neutral hydrogen in the PDR (dot-dashed line), and ionized hydrogen in the H II region (dotted line) for various central stars at the termination time. Bottom panel : The relative fraction of three masses presented in the middle panel. We plot $M \times M_{*}^{-2.3} \propto M \phi\left(M_{*}\right)$, where $\phi\left(M_{*}\right)$ is the IMF given by Miller \& Scalo (1979). In each panel, a positive feedback effect is expected in the unshaded mass range, while negative is expected in the shaded range.

First, the expanding shell finally dissolves due to the turbulent motion of the ambient medium. Ubiquitous supersonic turbulent motions in molecular clouds have been recognized by the observed broad emission line width. The corresponding velocity dispersion increases with the length scale as $\sigma \propto l^{1 / 2}$, and $\sigma \sim 2 \mathrm{~km} / \mathrm{s}$ at $l \sim 10 \mathrm{pc}$. We define the dissolving velocity, $v_{\mathrm{dlv}} \sim$ several $\times \mathrm{km} / \mathrm{s}$, and 
the shell dissolves at the time, $t_{\mathrm{dlv}}$, when the shell velocity becomes less than $v_{\mathrm{dlv}}$. Second, OB stars evolve to the Wolf-Rayet phase after the main-sequence phase, and finally explode as supernovae. Although the Wolf-Rayet winds and supernova explosions must cause the dynamical expansion of bubbles, we do not consider the evolution after the main-sequence lifetime, $t_{\mathrm{MS}}$. We evaluate the mass of the molecular gas in the shell at the termination time of the shell, $\min \left(t_{\mathrm{dlv}}, t_{\mathrm{MS}}\right)$. The top panel of Figure 1 presents $t_{\mathrm{dlv}}$ and $t_{\mathrm{MS}}$ with various central stars for $n_{\mathrm{H}}=100 \mathrm{~cm}^{-3}$ and $v_{\mathrm{dlv}}=2 \mathrm{~km} / \mathrm{s}$. As this panel shows, the dissolving time is shorter for the lower-mass star. This is because the dynamical time, $t_{\mathrm{dyn}}$ is shorter for the less luminous central star as $t_{\mathrm{dyn}} \equiv R_{\mathrm{st}} / C_{\mathrm{II}} \propto S_{\mathrm{UV}}^{1 / 3}$ (Paper II), where $S_{\mathrm{UV}}$ is the UV photon number luminosity. With shorter $t_{\mathrm{dyn}}$, the expansion quickly decelerates and terminates earlier. The main-sequence lifetime, $t_{\mathrm{MS}}$ is shorter for the higher-mass star, and the expansion is limited by $t_{\mathrm{MS}}$ for $M_{*} \gtrsim 30 M_{\odot}$.

The middle panel of Figure 1 presents the mass of the swept-up molecular gas at the termination time. This panel shows that $M_{\mathrm{sh}, \mathrm{m}}$ increases with the stellar mass, which verifies the triggering threshold condition. The molecular mass amounts to $M_{\mathrm{sh}, \mathrm{m}} \sim 10^{4-5} M_{\odot}$ for the massive central stars of $M_{*} \gtrsim 20 M_{\odot}$. In these cases, the $\mathrm{H}$ II region expands to $10-20$ pc by the termination of the shell. With the central star of $M_{*} \lesssim 20 M_{\odot}$, the accumulated molecular mass is significantly small. The shell dissolves before the accumulation of the molecular gas with the lower-mass star. To clarify the dominant feedback effect, we also plot the masses of the photodissociated and photoionized gas around the star at the same time. With the higher-mass stars of $M_{*} \gtrsim 20 M_{\odot}$, the accumulated molecular mass is much larger than both the ionized and photodissociated masses. Therefore, such massive stars give have a positive feedback effect on the clouds. With the lower-mass stars of $M_{*} \lesssim 20 M_{\odot}$, on the other hand, the photodissociated mass is much larger than the ionized and accumulated molecular masses. In these cases, the net feedback effect is negative, and the photodissociation by FUV photons is the dominant negative process, as argued by Diaz-Miller et al. (1998). The bottom panel of Figure 1 presents the masses multiplied by the population of exciting stars. When the higher-mass stars of $M_{*} \gtrsim 20 M_{\odot}$ are formed in the cloud, the positive feedback effect can dominate, despite a large number of lower-mass stars. Whether the net feedback effect is negative or positive depends on the mass of the most massive star in the cloud; positive for $M_{*} \gtrsim 20 M_{\odot}$, and vice versa in the current fiducial case.

\subsection{Threshold Stellar Mass for Starburst}

Using the calculated $M_{\mathrm{sh}, \mathrm{m}}$ for various central stars, we determine the threshold stellar mass, $M_{\text {thr }}$ for the burst of star formation. For example, in the fiducial case with $n_{\mathrm{H}}=100 \mathrm{~cm}^{-3}$ and $v_{\mathrm{dlv}}=2 \mathrm{~km} / \mathrm{s}$, the threshold mass calculated by equation (4) is $M_{\mathrm{thr}} \sim 18 M_{\odot}$ with $\epsilon=0.1$. This means that only a star more massive than $18 M_{\odot}$ can generate a shell massive enough so as to produce at least one star as massive as the original star.

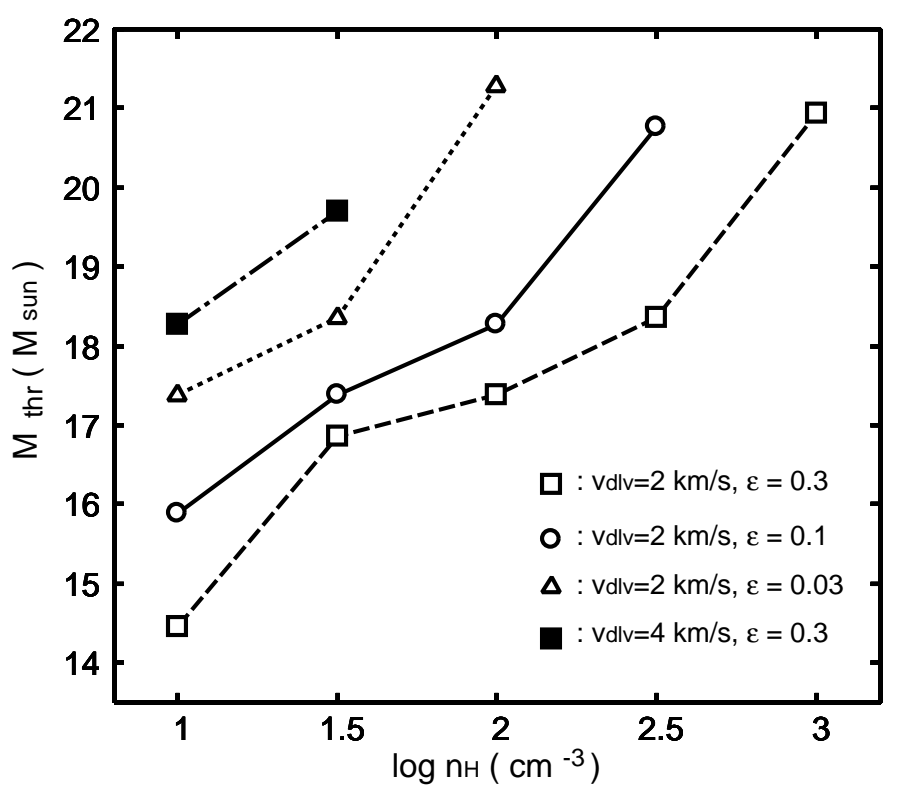

FIG. 2.- Threshold stellar masses, $M_{\mathrm{thr}}$ for the run-away triggering with different parameters. The horizontal axis is the ambient number density, and the different symbols denote different sets of dissolving velocity, $v_{\mathrm{dlv}}$ and star formation efficiency, $\epsilon$. Some symbols disappear at higher ambient densities, which means that the triggering threshold condition (eq.(5)) is not satisfied with the corresponding set of parameters. In these cases, triggering is not run-away, but a single event.

Note that the threshold mass is comparable to the stellar mass over which the character of feedback changes from negative to positive. In this case with $M_{*} \gtrsim 20 M_{\odot}$, therefore, the net feedback effect is positive, and strong enough to cause the run-away triggering.

We have examined the possibility of run-away triggering by calculating $M_{\text {thr }}$ with different parameters (Fig.2). For example, $M_{\mathrm{thr}}$ increases with decreasing SFE. This is because the more massive molecular shell is needed to compensate for the lower SFE. With $\epsilon=0.03$ in the fiducial case, we obtain $M_{\mathrm{thr}} \sim 21 M_{\odot}$. If the SFE is as low as $\epsilon \lesssim 0.01$, however, equation (4) is not satisfied with any $M_{\mathrm{thr}}$. This is because the increase in $M_{\mathrm{sh}, \mathrm{m}}$ grows saturated at about $\sim 10^{5} M_{\odot}$ as the mass of the central star increases (see Fig.1). Although the net feedback effect is positive with $M_{*} \gtrsim 20 M_{\odot}$, triggering is not run-away, but only a single event.

The threshold stellar mass also increases with the higher ambient density. In the denser ambient medium, the size of the H II region shrinks as $R_{\mathrm{st}} \propto n_{\mathrm{H}}^{-2 / 3}$. The total sweptup mass at the termination time also becomes smaller following the scaling relation, $M_{\mathrm{sh}} \propto n_{\mathrm{H}}^{-1}$, with the same central star. With the higher ambient density, therefore, $M_{\text {thr }}$ increases to produce the massive shell which enables the run-away triggering. Since the increase in $M_{\mathrm{sh}, \mathrm{m}}$ becomes saturated for the higher-mass stars, the run-away triggering becomes impossible unless the SFE is very high in the denser ambient medium. With $n_{\mathrm{H}}=10^{3} \mathrm{~cm}^{-3}$ and $v_{\mathrm{dlv}}=2 \mathrm{~km} / \mathrm{s}$, for example, an SFE as high as $\epsilon \sim 0.3$ is needed for the run-away triggering.

Finally, the higher dissolving velocity reduces the significance of triggering. The expanding shell dissolves earlier with higher $v_{\mathrm{dlv}}$, and the $\mathrm{H}$ II region sweeps up only the smaller region. Furthermore, the molecular fraction in the 
shell is preferentially reduced with the higher $v_{\mathrm{dlv}}$. During the dynamical expansion of the $\mathrm{H}$ II region, the column density of the shell, $N_{\mathrm{sh}}$ increases as $N_{\mathrm{sh}} \sim n_{\mathrm{H}} R_{\mathrm{IF}}(t) / 3$. Therefore, the smaller H II region has a shell with lower column density, though the incident FUV radiation is strong owing to the poor geometrical dilution. Since the FUV radiation shielding is inefficient, it is hard for molecules to accumulate in the shell. With $v_{\mathrm{dlv}}=4 \mathrm{~km} / \mathrm{s}$ and $\epsilon=0.3$, for example, the run-away triggering is possible only with the low ambient density of $n_{\mathrm{H}} \lesssim 10^{1.5} \mathrm{~cm}^{-3}$.

\section{DISCUSSIONS AND CONCLUSIONS}

In this letter, we suggest a possible mode of selfpropagating star formation in molecular clouds, which should be examined in further studies. Although we have adopted the homogeneous ambient density, for example, real clouds show clumpy structure with a turbulent velocity field. Recently, Mellema et al. (2006) have calculated the dynamical expansion of the H II region in turbulent molecular clouds. They have shown that the clumpy medium leads to an irregular ionization front. The radial position of the ionization front approximately agrees with that in the homogeneous medium on average with the mean density, but significantly depends on the radial direction. More theoretical studies and multi-dimensional numerical simulations of the deformation and fragmentation processes of the swept-up shell are needed. Observational estimation of the SFE in the shell might be interesting and useful for the present model.

The stellar wind from the massive star is another omission in our evaluation. The expanding wind-driven bubble can modify the dynamics of the ISM around massive stars. When the bubble pressure is much higher than the ambient pressure, the time evolution of the bubble size and pressure is given by,

$$
\begin{aligned}
R_{\mathrm{b}}(t) & =\left(\frac{125}{154 \pi}\right)^{1 / 5} L_{\mathrm{w}}^{1 / 5} \rho_{0}^{-1 / 5} t^{3 / 5}, \\
P_{\mathrm{b}}(t) & =\frac{7}{(3850 \pi)^{2 / 5}} L_{\mathrm{w}}^{2 / 5} \rho_{0}^{3 / 5} t^{-4 / 5},
\end{aligned}
$$

where $L_{\mathrm{w}}$ is the wind mechanical luminosity (Weaver et al. 1977). Here, we evaluate the significance of the winddriven bubble by comparing the ratio between the bubble pressure and H II pressure $\left(P_{\mathrm{II}}=\rho_{0} C_{\mathrm{II}}^{2}\right)$ at $R_{\mathrm{b}}=R_{\mathrm{st}}$. With equations (6) and (7),

$$
\left.f_{\mathrm{P}} \equiv \frac{P_{\mathrm{b}}}{P_{\mathrm{II}}}\right|_{R_{\mathrm{b}}=R_{\mathrm{st}}}=\frac{10}{11}\left(\frac{77}{250}\right)^{1 / 3} f_{\mathrm{L}}^{2 / 3} \sim\left(\frac{f_{\mathrm{L}}}{2}\right)^{2 / 3}
$$

where we define $f_{\mathrm{L}} \equiv L_{\mathrm{w}} / L_{\mathrm{st}}$ and $L_{\mathrm{st}} \equiv 4 \pi R_{\mathrm{st}}^{2} \rho_{0} C_{\mathrm{II}}^{3}$ (McKee et al. 1984). If $f_{\mathrm{P}} \lesssim 1$, the bubble is confined by the ambient H II pressure before reaching the initial Strömgren radius. The numerical value of $f_{\mathrm{L}}$ can be estimated by $f_{\mathrm{L}}=0.8\left(S_{\mathrm{UV}} / 10^{49} \mathrm{~s}^{-1}\right)^{5 / 6} n_{\mathrm{H}}^{1 / 3}$ (Abbott 1982). With the number density of $n_{\mathrm{H}}=100 \mathrm{~cm}^{-3}, f_{\mathrm{P}} \sim 0.4$ for a $20 M_{\odot}$ star and $f_{\mathrm{P}} \sim 1.5$ for a $50 M_{\odot}$ star. Therefore, the stellar wind only slightly increases the initial pressure of the H II region with the star of $M_{*} \lesssim 50 M_{\odot}$. The effect of the wind-driven bubble becomes significant with a very massive star $\left(\sim 100 M_{\odot}\right)$, highly clustered massive stars, or in higher density media. When a wind-driven bubble dominates the dynamics, the bubble sweeps up the larger region at higher velocity than the expansion only due to the $\mathrm{H}$ II region overpressure. This situation may even promote the positive feedback effect, and should be separately studied in detail.

Finally, we summarize the results. First, we have formulated the conditions for the positive feedback process by which the triggered star formation continues in the sweptup shell. The threshold stellar mass, $M_{\mathrm{thr}}$, is defined as the mass of the central star that sweeps up the molecular gas in the shell, where at least one new star with mass $M_{*}=M_{\mathrm{thr}}$ forms after the shell fragmentation. In order to evaluate $M_{\mathrm{thr}}$, we have calculated how massive molecular shells can form around various central stars. In the fiducial case with $n_{\mathrm{H}}=100 \mathrm{~cm}^{-3}$ and $v_{\mathrm{dlv}}=2 \mathrm{~km} / \mathrm{s}$, the swept-up molecular mass increases with the stellar mass, and amounts to $M_{\mathrm{sh}, \mathrm{m}} \sim 10^{4-5} M_{\odot}$ with the massive star of $M_{*} \gtrsim 20 M_{\odot}$. We have also calculated the photoionized and photodissociated masses to clarify the net feedback effect. The negative effect of photodissociation is important with the lower-mass star of $M_{*} \lesssim 20 M_{\odot}$, but is dominated by the positive effect with the higher-mass star. We have calculated the threshold mass as $M_{\mathrm{thr}} \sim 18 M_{\odot}$ in the fiducial case with $\epsilon=0.1$. We have also calculated $M_{\text {thr }}$ with different parameters, and examined the generality of run-away triggering. We have found $M_{\mathrm{thr}} \sim 15-20 M_{\odot}$ in various situations. The triggering process is only a single event with the higher ambient density, higher dissolving velocity, or lower SFE.

We thank a anonymous referee for fruitful comments that improved the presentation of the paper. SI is supported by the Grant-in-Aid $(15740118,16077202)$ from the Ministry of Education, Culture, Sports, Science, and Technology (MEXT) of Japan.

\section{REFERENCES}

Abbott, D.C. 1982, ApJ, 259, 282

Deharveng, L., Lefloch, B., Zavagno, A., Caplan, J., Whitworth, A.P., Nadeau, D. \& Martin, S. 2003, A\&A, 408, 25L

Deharveng, L., Zavagno, A. \& Caplan, J. 2005, A\&A, 433, 565

Diaz-Miller, R.I., Franco, J. \& Shore, S.N. 1998, ApJ, 501, 192

Elmegreen, B.G. \& Lada, C.J. 1977, ApJ, 214, 725

Elmegreen, B.G. 1989, ApJ, 340, 786

Evans, N.J.II, 1999, ARA\&A, 37, 311

Franco, J., Shore, S.N. \& Tenorio-Tagle, G. 1994, ApJ, 436, 795

Hosokawa, T. \& Inutsuka, S. 2005, ApJ, 623, 917 (Paper I)

Hosokawa, T. \& Inutsuka, S. 2006, ApJ, 646, 240 (Paper II)

Keto, E., Ho, L.C. \& Lo, K.Y. 2005, ApJ, 635, 1062
Lada, C.J. \& Lada, E.A. 2003, ARA\&A, 41, 57

Matzner, C.D. 2002, ApJ, 566, 302

McKee, C.F., van Buren, D. \& Lazareff, B. 1984, ApJ, 278, 115L

Mellema, G., Arthur, S.J., Henney, W.J., Iliev, I.T. \& Shapiro, P.R. 2006, submitted to ApJ (astro-ph/0512554)

Miller, G.E. \& Scalo, J.M. 1979, ApJS, 41, 513

Weaver, R., McCray, R., Castor, J., Shapiro, P. \& Moore, R. 1977, ApJ, 218, 377

Whitworth, A. 1979, MNRAS, 186, 59

Williams, J.P. \& McKee, C.F. 1997, ApJ, 476, 166

Zavagno, A., Deharveng, L., Comeron, F., Brand, J., Massi, F., Caplan, J. \& Russeil, D. 2005, A\&A, 446, 171 